Case Report

\title{
Development of Acute Inflammatory Demyelinating Polyneuropathy 11 Days after Spinal Surgery: A Case Report and Literature Review
}

\author{
Eiichi Kakehi ${ }^{D},{ }^{1}$ Tadataka Kawakami, ${ }^{2}$ Yukiko Ishikawa, ${ }^{3}$ Takashi Matsuoka, ${ }^{4}$ \\ Naoki Nakagawa, ${ }^{5}$ Tugutake Morishita, ${ }^{5}$ Shohei Taniguchi, ${ }^{1}$ Yukinobu Akamatsu, \\ Shigehisa Sakurai, ${ }^{1}$ Akane Hirotani, ${ }^{1}$ Takafumi Nozaki, ${ }^{1}$ Keisuke Shoji, ${ }^{1}$ Seiji Adachi, ${ }^{1}$ \\ Kazuhiko Kotani, ${ }^{3}$ and Masami Matsumura ${ }^{3}$ \\ ${ }^{1}$ Department of General Medicine, Tottori Municipal Hospital, Tottori-City, Tottori, Japan \\ ${ }^{2}$ Department of Neurology, Shin-Oyama City Hospital, Oyama-City, Tochigi, Japan \\ ${ }^{3}$ Center for Community Medicine, Jichi Medical University, Shimotsuke-City, Tochigi, Japan \\ ${ }^{4}$ Department of Neurology, Tottori Municipal Hospital, Tottori-City, Tottori, Japan \\ ${ }^{5}$ Department of Neurological Surgery, Tottori Municipal Hospital, Tottori-City, Tottori, Japan
}

Correspondence should be addressed to Eiichi Kakehi; openwater_swimming5933@yahoo.co.jp

Received 16 May 2021; Revised 17 July 2021; Accepted 19 July 2021; Published 28 July 2021

Academic Editor: Mamede de Carvalho

Copyright (C) 2021 Eiichi Kakehi et al. This is an open access article distributed under the Creative Commons Attribution License, which permits unrestricted use, distribution, and reproduction in any medium, provided the original work is properly cited.

Guillain-Barré syndrome (GBS) usually has a good prognosis; however, patients may develop sequelae without prompt treatment. We herein describe an 81-year-old woman who developed acute-onset excruciating thigh pain and weakness in her lower extremities after spinal surgery. We diagnosed acute inflammatory demyelinating polyradiculoneuropathy by a nerve conduction study, which showed findings of demyelination without cerebrospinal fluid analysis because of a spinal prosthesis. Although antiGM1 and anti-GalNAc-GD1a antibodies were positive, the patient was clinically diagnosed with acute inflammatory demyelinating polyradiculoneuropathy (a subtype of GBS), not acute motor axonal neuropathy. She recovered well with immunoglobulin therapy. A literature review of 18 cases revealed that unexplained weakness, areflexia, and numbness of the extremities after spinal surgery, a shorter time from spinal surgery to symptom onset to general GBS, abnormal nerve conduction study results, normal spinal imaging findings, and the development of atypical symptoms such as cranial and autonomic nerve syndrome and respiratory failure are useful for diagnosing GBS when cerebrospinal fluid examination cannot be performed after spinal surgery.

\section{Introduction}

Guillain-Barré syndrome (GBS) is an acute autoimmune inflammatory polyneuropathy that affects the peripheral nervous system and is usually triggered by infectious disease [1]. The incidence of GBS in the general population of the United States is reportedly $1-2$ per 100,000 [2]. The postsurgical incidence of GBS is 4.1 per 100,000 patients, and the incidence after spinal surgery is 1 per 2,000 patients [3]. The onset of complications after spinal surgery ranges from $4 \%$ to $19 \%$ [4], and the overall incidence of neurologic complications is $<1 \%$ [5]. The mortality rate of GBS within 1 year after diagnosis is reportedly $4.4 \%$ [6], and the rate of requiring mechanical ventilation is $38 \%$ [7].

The early symptoms of GBS mimic an acute spinal cord compression disorder or nerve root compression syndrome, and GBS can thus be difficult to differentiate from complications after spinal surgery. Cerebrospinal fluid (CSF) 
examination is essential for diagnosing GBS; however, postoperative spinal changes often make CSF collection and analysis difficult.

Although GBS is a rare complication after spinal surgery, its correct diagnosis is crucial because it will prevent a reoperation based on a misdiagnosis of recurrent spinal problems. We herein describe a case of GBS that mimicked spinal cord injury after spinal surgery. Although the characteristics of GBS after spinal surgery have been previously reported [8], the strategies for differentiating postoperative complications and GBS have not been well summarized. We reviewed previous reports to clarify these diagnostic challenges.

\section{Case Presentation}

An 81-year-old Japanese woman with a history of cervical canal stenosis and bilateral knee osteoarthritis visited our outpatient orthopedics department with the chief complaint of claudication and pain in the back of the thigh. These symptoms had been present for 1 year and had worsened 3 months before her visit. She had developed no recent infections nor received any vaccinations in the previous few months, and she had no surgical history. Muscle strength testing according to the Medical Research Council scale demonstrated grade 5/5 muscle strength in the bilateral upper and lower extremities, but her bilateral extensor digitorum longus and brevis strength was 4/5. Her bilateral patellar and Achilles tendon reflexes were brisk. On sensory examination, she showed hyperesthesia in the bilateral distal part of lower legs on light touch testing. Magnetic resonance imaging (MRI) showed severe lumbar spinal stenosis at L1-5 and spondylolisthesis at L4/5 (Figure 1(a)). She was diagnosed with spondylolisthesis with lumbar spinal stenosis. An L1-5 laminectomy and L4/5 posterolateral fusion were performed under general anesthesia without surgical complications. Her claudication improved, and she was able to ambulate with a walker. She developed no acute complications for 8 days after the surgery.

Nine days after the surgery, she developed only one episode of transient soft stool without fever, abdominal pain, nausea, or vomiting. Eleven days after the surgery, she reported acute onset of excruciating bilateral thigh pain. She subsequently developed difficulty walking because of ascending weakness of her bilateral lower extremities. The tingling in her bilateral lower extremities was exacerbated. On physical examination, she was conscious and afebrile, and her surgical wound was healing well. All cranial nerves were intact, and she had no difficulty swallowing or breathing. Muscle strength testing showed grade 4/5 in the upper distal extremities and $3 / 5$ in the lower distal and proximal extremities, with no muscle atrophy. On sensory examination, the hyperesthesia in the bilateral distal parts of her lower legs was exacerbated on light touch testing. The bilateral tendon reflexes of the biceps brachii, triceps brachii, and brachioradialis were normal, but the bilateral patellar and Achilles tendon reflexes were absent. Pathological reflexes, including those in the upper and lower extremities, were negative. She was unable to stand or walk. Routine blood tests, including a complete blood count, glucose, electrolytes, renal function, and liver enzymes, were within normal limits. Serum anti-GM1-IgG and anti-GalNAcGD1a-IgG antibodies were positive, with optical density values (control $<0.1$ ) of 0.157 and 0.135 , respectively.

Spinal computed tomography showed no evidence of spinal cord compression (Figures 1(b) and 1(c)). Brain MRI showed no abnormalities. MRI of the whole spine showed moderate disc herniation at the $\mathrm{C} 3 / 4$ level without significant cord or nerve root compression or an epidural hematoma or abscess (Figures 1(d) and 1(e)). An acute polyradiculoneuropathy was suspected based on rapidly progressive ascending weakness, sensory disturbance, and areflexia of the lower extremities without implant-related complications. We therefore consulted a neurologist, and a nerve conduction study (NCS) was performed 21 days after surgery. Table 1 and Figure 2 show the results of the NCS. The median and tibial motor nerves showed findings of demyelination [9], such as prolonged terminal latencies, reduction in the compound muscle action potential, a slowed conduction velocity, and temporal dispersion. The ulnar motor nerve showed a decrease in amplitude. The F wave was absent in the bilateral median, ulnar, and tibial motor nerves. The sensory examination showed an abnormal median and normal sural sensory response [10]. Because we did not perform needle electromyography, therefore, we could not evaluate fibrillation potentials. In addition, we did not identify visible fasciculation in any involved muscles or any new central nerve disorders using neuroimaging studies. A CSF examination was difficult to perform because the patient had recently undergone lumbar spinal surgery. Given the acute onset of her symptoms, normal spinal imaging findings, and significant evidence of demyelination on the NCS, she was diagnosed with GBS.

Treatment was started 9 days after the appearance of symptoms. Immunoglobulin therapy was started at a dose of $0.4 \mathrm{~g} / \mathrm{kg}$ for 5 days. After 1 month of follow-up, her upper and lower extremity weakness resolved, and motor examination showed grade 5/5 in the upper extremities and 4/5 in the lower extremities. She had residual tingling in both lower extremities. An NCS performed 38 days after surgery showed that the median and ulnar motor nerve compound muscle action potentials had residual prolonged terminal latencies but improved amplitudes. There was no improvement in the tibial nerve with temporal dispersion on day 21 and day 38. The median and ulnar sensory nerve abnormalities were exacerbated, but the sural nerve remained normal (Table 1, Figure 2). She needed a cane to walk, but muscle strength testing according to the Medical Research Council scale showed that her extremity muscle strength had improved to grade $5 / 5$. She was able to ambulate with a cane and was subsequently transferred to a rehabilitation hospital.

\section{Literature Review}

To understand the clinical characteristics of our case, we reviewed case reports of GBS after spinal surgery. A search of the PubMed database was performed using the terms 


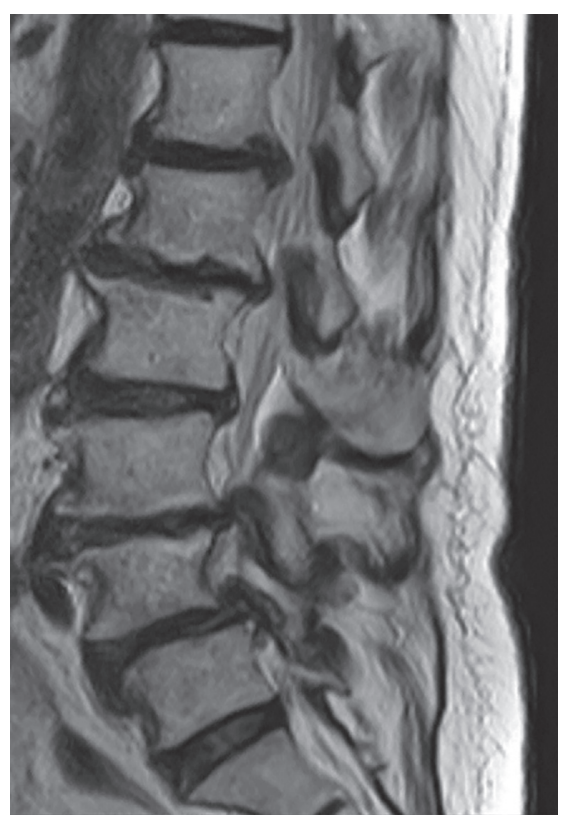

(a)

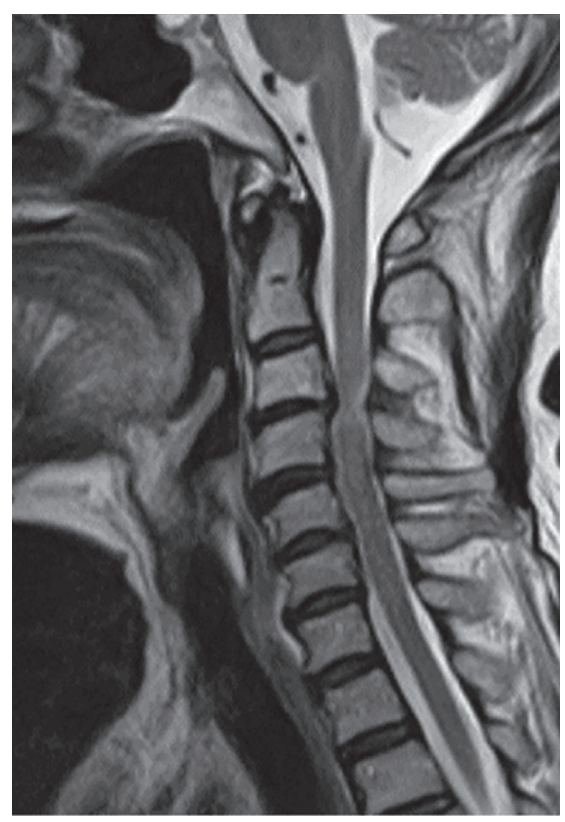

(d)
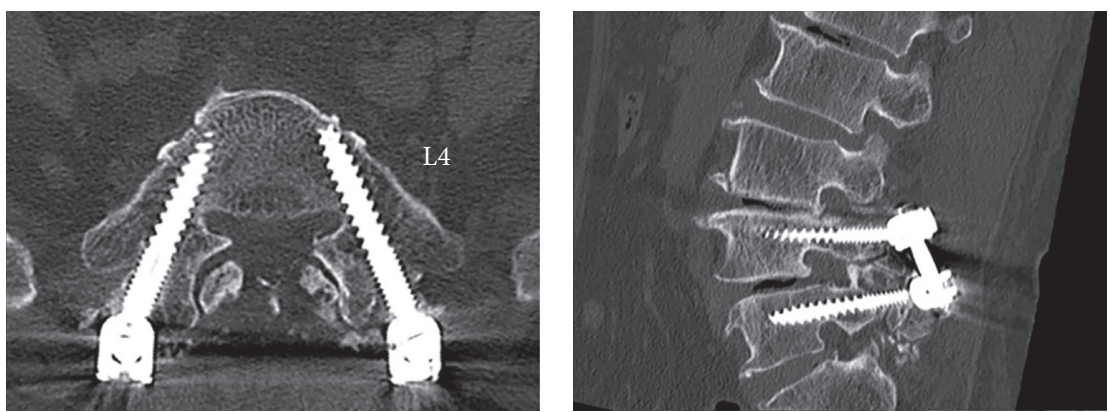

(b)

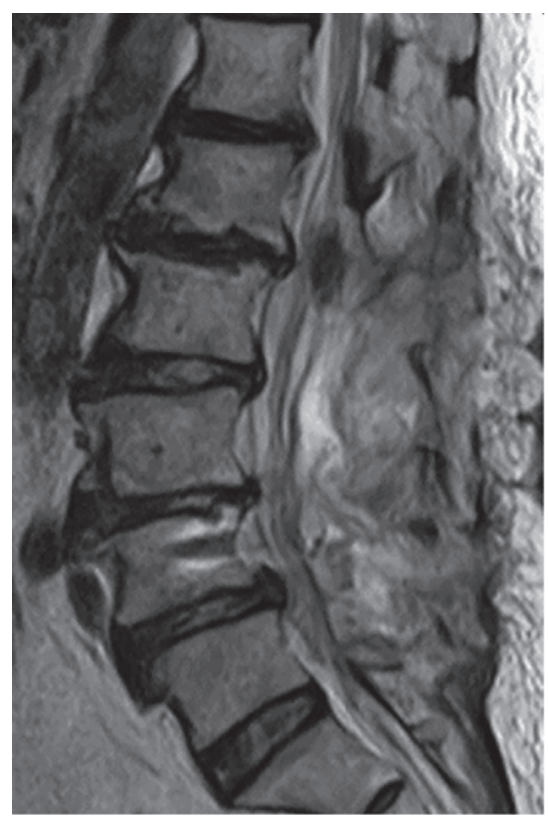

(e)

FIgURE 1: Imaging findings. (a) Lumbar magnetic resonance imaging (MRI) demonstrating severe lumbar spine stenosis at L1-5 and spondylolisthesis at L4/5. (b), (c) No percutaneous pedicle screw deviation or compression observed in the spinal canal. (d) Cervical MRI revealing spinal canal stenosis extending to C4-6 and disc herniation at the C3/4 level. (e) Lumbar MRI showing bilateral foraminal narrowing at the L4/5 level after laminectomy for L4 anterior spondylolysis, but no apparent implant deviation.

"Guillain-Barré syndrome" AND "spinal surgery OR spine surgery" for all articles published until May 2021. We identified 14 published reports involving 18 patients with GBS after spinal surgery (Table 2) [11-24].

Table 2 summarizes the characteristics of previously described patients diagnosed with GBS after spinal surgery. Their average age was 56.8 years (range, 33-73 years), and the male/female ratio was 11/7 [11-24]. The vertebral regions affected by the surgery were the lumbar region in eight
(44.4\%) patients $[16,17,20-24]$, the cervical region in four $(22.2 \%)[15,18]$, the thoracolumbar region in three $(16.7 \%)$ $[11,14,18]$, the thoracic region in one $(5.6 \%)[13]$, the thoracosacral region in one (5.6\%) [11], and the lumbosacral region in one (5.6\%) [19]. The average duration of time from spinal surgery to the onset of GBS symptoms was 7.4 days (range, 1 hour to 22 days), with two patients having a duration of $\leq 3$ hours [17], and the duration of time from onset of GBS symptoms to diagnosis was 7.2 days (range, 
TABLE 1: Electrophysiological findings 21 and 38 days after spinal surgery.

\begin{tabular}{|c|c|c|c|c|c|c|c|c|c|c|c|c|c|c|c|c|c|}
\hline & \multirow[t]{3}{*}{ Site } & \multicolumn{4}{|c|}{$\begin{array}{c}\text { Terminal latency }(\mathrm{ms}) \\
\text { motor: median }<3.7 \text {, ulnar } \\
<2.9 \text {, tibial }<5.1 \text {, sensory: } \\
\text { median }<2.9 \text {, ulnar }<2.6 \text {, } \\
\text { sural }<3.1\end{array}$} & \multicolumn{4}{|c|}{$\begin{array}{l}\text { Amplitude }(\text { motor }=\mathrm{mV} \text {, } \\
\text { sensory }=\mu \mathrm{V}) \text {, motor: } \\
\text { median }>4.9 \text {, ulnar }>5.5 \text {, } \\
\text { tibial }>10.1 \text {, sensory: median } \\
\text { and ulnar }>29.4 \text {, sural }>3.3\end{array}$} & \multicolumn{4}{|c|}{$\begin{array}{l}\text { Conduction velocity }(\mathrm{m} / \mathrm{s}) \text {, } \\
\text { motor: median }>58 \text {, ulnar } \\
>59 \text {, tibial }>48 \text {, sensory: } \\
\text { median }>65 \text {, ulnar }>64 \text {, sural } \\
>46\end{array}$} & \multicolumn{4}{|c|}{ FCV $(\mathrm{m} / \mathrm{s})$, median $>69$} \\
\hline & & \multicolumn{2}{|c|}{ Day 21} & \multicolumn{2}{|c|}{ Day 38} & \multicolumn{2}{|c|}{ Day 21} & \multicolumn{2}{|c|}{ Day 38} & \multicolumn{2}{|c|}{ Day 21} & \multicolumn{2}{|c|}{ Day 38} & \multicolumn{2}{|c|}{ Day 21} & \multicolumn{2}{|c|}{ Day 38} \\
\hline & & Right & Left & Right & Left & Right & Left & Right & Left & Right & Left & Right & Left & Right & Left & Right & Left \\
\hline \multicolumn{18}{|l|}{ Motor } \\
\hline Med & rist & 20.0 & 6.6 & 20.1 & 17.8 & 0.87 & 1.37 & 3.21 & 273 & 35.6 & 31.0 & 38.3 & 33.9 & NR & NR & 44.3 & 36.5 \\
\hline $\mathrm{U}$ & Wrist & 4.90 & 4.65 & 5 & 5.30 & 2.2 & 3.5 & 4.0 & 5. & 41.0 & 50.6 & 34.3 & 42.9 & NR & NR & NR & NR \\
\hline Tibial & $\mathrm{Ar}$ & 6.10 & 9.60 & 7.80 & 11.20 & 1.70 & 0.83 & 1.6 & 0.95 & 34.5 & 27.2 & 30.1 & 34.4 & NR & NR & 36.9 & 48.7 \\
\hline \multicolumn{18}{|l|}{ Sensory } \\
\hline Median & & NR & & $\mathrm{NH}$ & & $\mathrm{N}$ & & & & $\mathrm{N}$ & NF & NR & NR & - & - & - & - \\
\hline Ulnar & Wrist & 2.46 & 2.4 & 2.66 & - & 13.20 & 16.50 & 10.70 & 13.10 & 67.5 & 51.3 & 53.1 & 53.9 & - & - & - & - \\
\hline Sural & Midcalf & 2.70 & 3.00 & 2.60 & 3.10 & 7.40 & 3.30 & 8.10 & 6.40 & 51.1 & 46.7 & 53.8 & 45.2 & - & - & - & - \\
\hline
\end{tabular}

FCV, F wave conduction velocity; NR, no response.

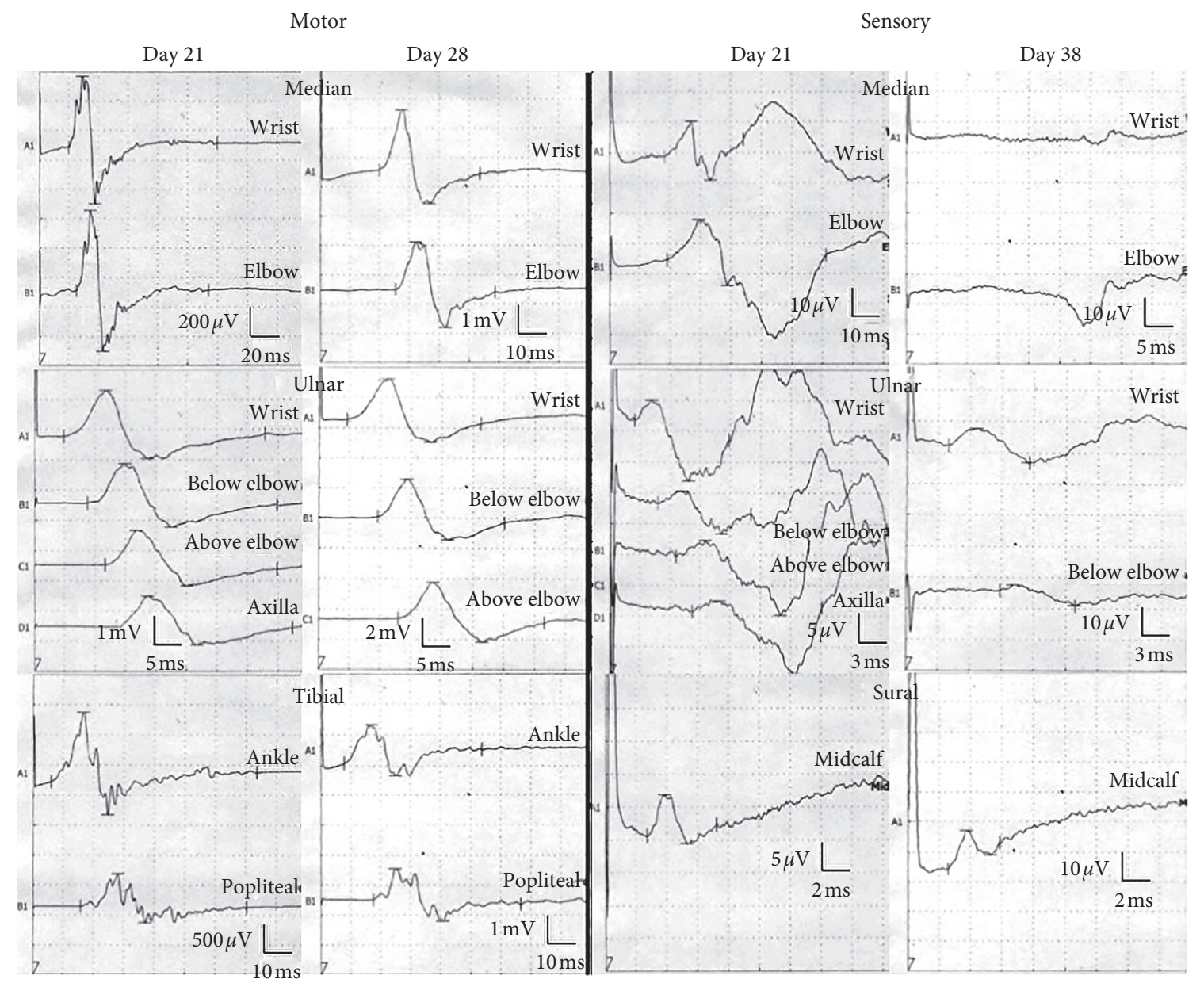

Figure 2: Nerve conduction study results. At 21 days after surgery, the median and tibial motor nerves showed findings of demyelination, such as prolonged terminal latencies, reduction in the compound muscle action potential, a slowed conduction velocity, and temporal dispersion. The ulnar motor nerve showed a decrease in amplitude. The sensory examination showed an abnormal median and normal sural sensory response. The $\mathrm{F}$ wave was absent in the bilateral median, ulnar, and tibial motor nerves (data not shown). At 38 days after surgery, the median and ulnar motor nerve compound muscle action potentials had residual prolonged terminal latencies but improved amplitudes. The tibial nerve showed temporal dispersion on both day 21 and day 28 with no improvement. The median and ulnar sensory nerves abnormalities were exacerbated, but the sural nerve remained normal. 


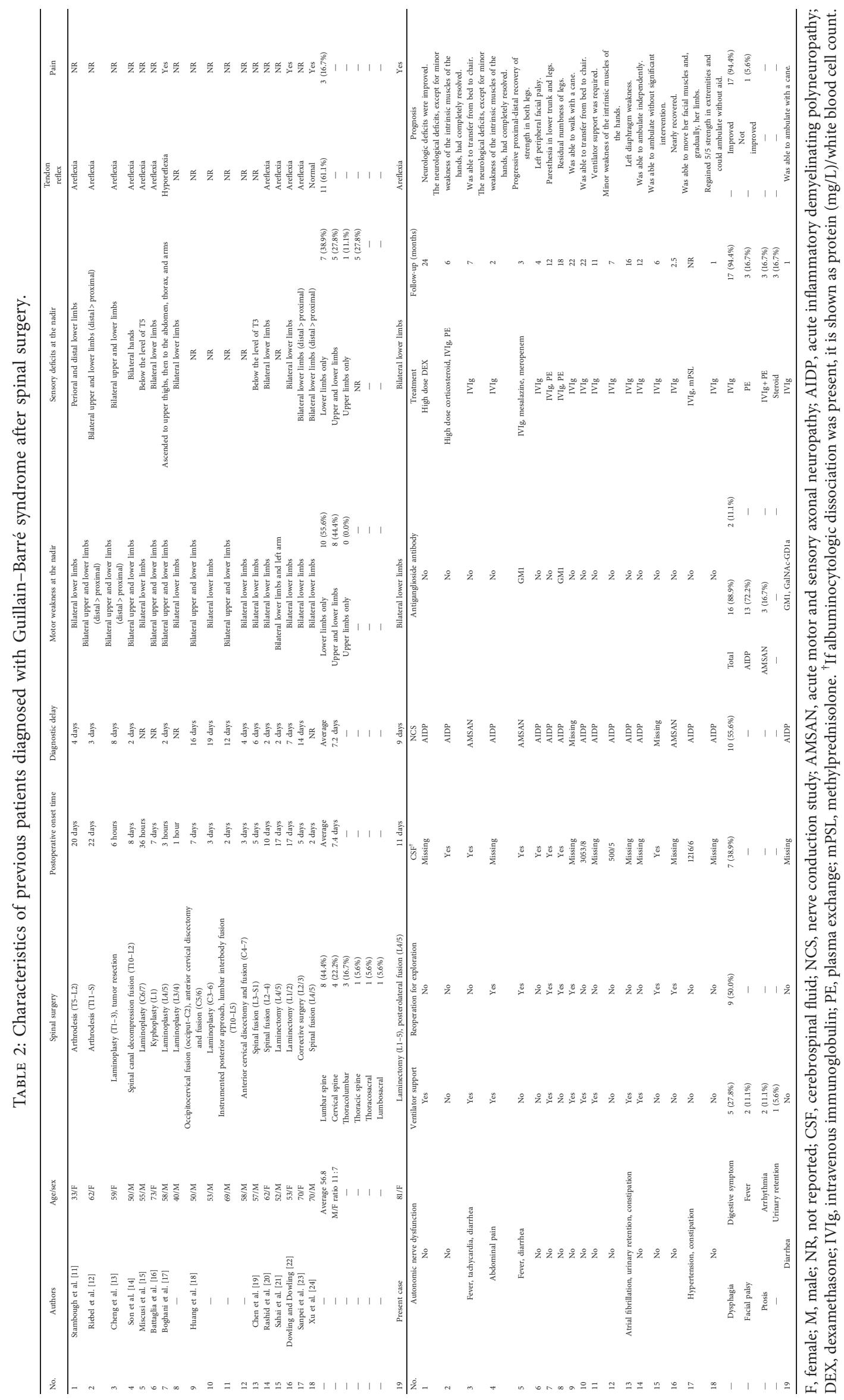


1-19 days). Ten (55.6\%) patients had motor weakness at the nadir in the lower limbs only [11, 15, 17-20, 22-24], and eight $(44.4 \%)$ had motor weakness in the upper and lower limbs [12-14, 16-18, 21]. Seven (38.9\%) patients had sensory deficits at the nadir in the lower limbs only $[11,16,17,20,22-24]$, five $(27.8 \%)$ in the upper and lower limbs $[12,13,15,17,19]$, and one (11.1\%) in the upper limbs only [14]. Eleven (61.1\%) patients showed areflexia or hyporeflexia [11-17, 20-23]. Three (16.7\%) patients reported pain $[17,22,24]$. Nine $(50.0 \%)$ patients had cranial nerve symptoms $[13,14,16,18,19,23,24]$, which included facial paralysis, dysphagia, decreased gag reflex, and impaired vocalization. Five $(27.8 \%)$ patients had autonomic nerve symptoms [13-15, 19, 23], which included fever, tachycardia, atrial fibrillation, urinary retention, abdominal pain, diarrhea, and constipation. Nine (50.0\%) patients needed mechanical ventilator support by intubation $[11,13,14,17-20]$, one patient could not be weaned [18], and the rest received temporary support. Seven (38.9\%) patients needed a reoperation to determine the cause of postoperative complications $[14,15,17,18]$. CSF examinations were performed in $10(55.6 \%)$ patients, 6 of $12(50.0 \%)$ patients who underwent lumbar surgery $[12,16,17,21,23]$, and 4 of $5(80.0 \%)$ patients who underwent nonlumbar surgery $[13,15,18])$. Fifteen (88.2\%) patients underwent an NCS [11-20,22-24]. Antiganglioside antibodies were detected in two patients $[15,17]$, but the prior infection status was unknown. Seventeen patients underwent intravenous immunoglobulin (IVIg) therapy [12-24], three patients received concomitant plasma exchange (PE) therapy $[12,17]$, and two patients received concomitant high-dose corticosteroids [12, 23]. One patient received high-dose corticosteroids only [11]. No deaths occurred. The motor impairment was improved in many patients; however, some had residual sensory disturbances.

\section{Discussion}

Clinicians should be aware of progressive muscle weakness, areflexia, or numbness after a spinal operation as potential signs of GBS. The clinical features that are helpful for the diagnose of GBS after spinal surgery can be summarized from previous literature as follows: shorter time from spinal surgery to symptom onset to general GBS, abnormal NCS results, normal spinal imaging, and the development of atypical symptoms such as cranial and autonomic nerve syndrome and respiratory failure. We consider that even when CSF testing is not available, an NCS is preferable to CSF analysis in the diagnosis of GBS and is clinically useful. Furthermore, rapid initiation of treatment with IVIg and/or $\mathrm{PE}$ is useful for improving the prognosis [7]. GBS after spinal surgery presents a unique diagnostic challenge, and we devised a flowchart (Figure 3) based on previous literature.

The mechanism of GBS development most likely involves a recent preceding upper or lower respiratory tract infection or gastrointestinal illness followed by an immunological response [25]. The mechanism by which GBS develops after surgery is considered to involve an interaction between the anesthetic agents and peripheral nerve myelin or local trauma to roots; this may initiate a cascade of immunologic events that result in demyelinating neuropathy [26]. Staff et al. [27] reported an inflammatory change in nerve biopsy samples from patients with nontraumatic postoperative neuropathy within 30 days after surgery. Surgery alters the balance of the immune system and causes transient immunosuppression, which can trigger the onset of GBS and promote subclinical infection that can also predispose a patient to GBS $[28,29]$.

Serum anti-GM1 and anti-GalNAc-GD1a antibodies, which were detected in our case, are reportedly associated with acute motor axonal neuropathy in electrophysiological studies due to preinfection by Campylobacter jejuni $[30,31]$. Ogawa et al. [32] reported that the presence of anti-GM1/ GalNAc-GD1a antibodies was correlated with pure motor GBS characterized by antecedent respiratory infection, fewer cranial nerve deficits, and conduction blocks at intermediate sites of motor nerves. We did not perform serological testing for C. jejuni because our patient had only one episode of soft stool, had not ingested any components that could cause infectious enteritis, and was not considered to have Campylobacter enteritis. Therefore, the relationship between the antiganglioside antibodies and the patient's symptoms was unclear.

Most reported cases of postoperative GBS occurred within 1-3 weeks, with a maximum of 6 weeks [3]. Our literature review indicated that the onset of GBS symptoms after spinal surgery tended to be earlier than that after nonspinal surgery, although it was later than the occurrence of other complications such as iatrogenic spinal cord injury or postoperative herniation [19]. However, Wakerley and Yuki [33] reported that the onset of GBS within 2 days after surgery would be triggered not by the surgery but by preoperative triggers and subsequent immune mechanisms (e.g., infection, vaccines, and trauma). Xu et al. [24] reported that a patient developed GBS 2 days after surgery and had received an influenza vaccine 10 days before surgery. The duration of postoperative symptom onset and a careful preoperative history can help to reveal the cause.

An NCS is the most useful examination for GBS after spinal surgery because an NCS is less likely to be affected by the postoperative state than is a CSF analysis [19]. An NCS in patients with GBS typically shows a multifocal demyelinating process, including conduction block or temporal dispersion in motor nerves [25]. Abnormal median and normal sural sensory responses are highly suggestive of acute inflammatory demyelinating polyneuropathy (AIDP) subtypes of GBS [10]. Our patient exhibited these findings, which were useful for diagnosing the underlying cause of her muscle weakness. Uncini and Kuwabara proposed the characteristics of "nodopathy" as rapidly reversible nerve conduction block due to paranodal myelin detachment and nodal sodium channel disruption, the absence of excessive temporal dispersion associated with remyelination, and eventual complement-mediated axonal degeneration depending on the severity [34]. In the present case, the findings of temporal dispersion of the tibial nerve at day 38 (after the symptoms had improved) and no evidence of conduction block support the diagnosis of AIDP rather than 


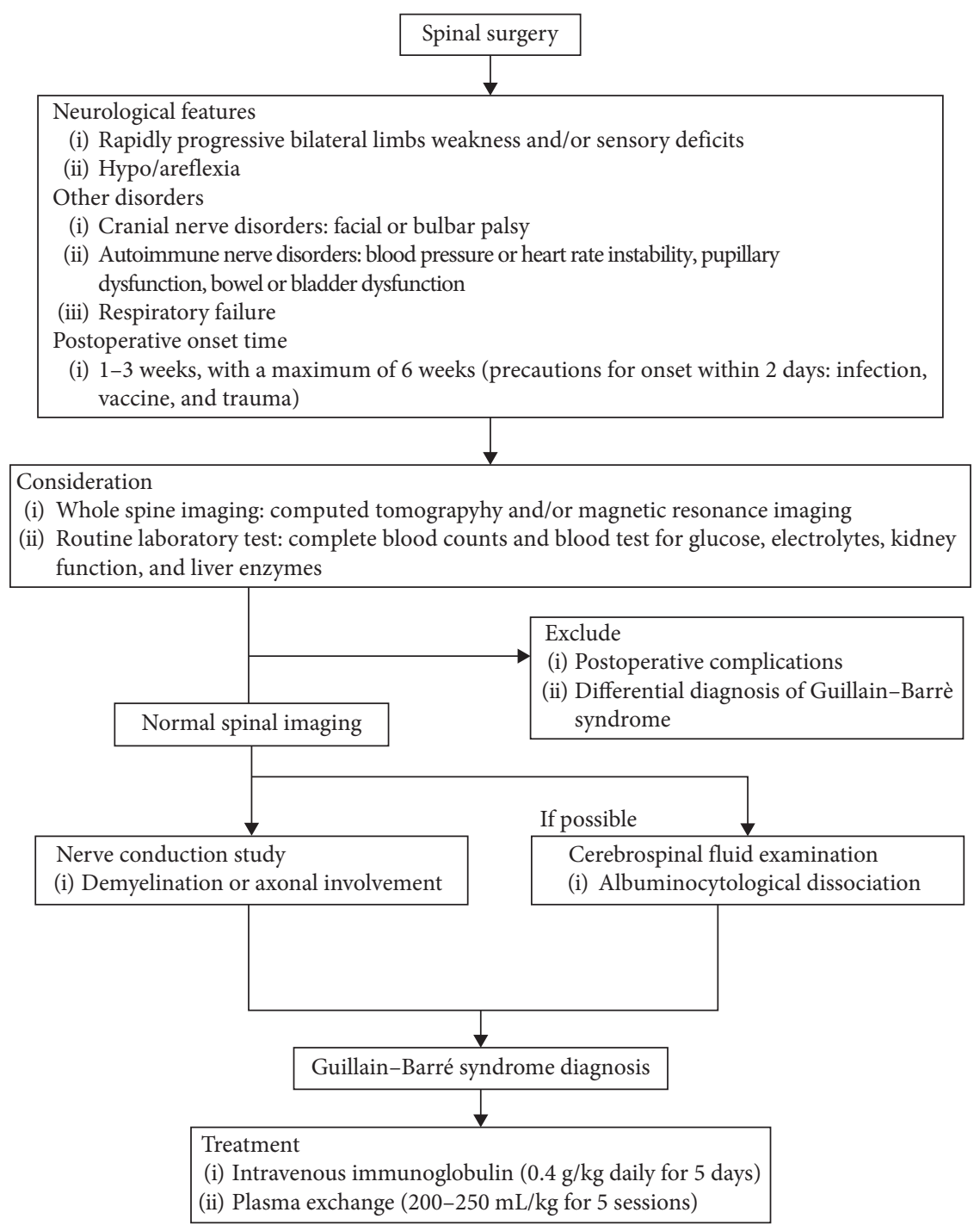

FIGURE 3: Strategies for diagnosis and treatment of Guillain-Barré syndrome after spinal surgery.

nodopathy. Anti-GM1 antibodies have been reported to be associated with acute motor axonal neuropathy, but our literature review revealed an association between acute motor and sensory axonal neuropathy (AMSAN) [15] and AIDP [17]. Our literature review also showed that AMSAN has recently been associated with spinal surgery $[13,15,22]$. AMSAN is rare and usually presents with severe symptoms over a short period, and patients often experience prolonged and incomplete recovery compared with other forms of GBS. The maximum incidence of electrophysiological abnormalities is reportedly 4-12 weeks after the onset of neurological symptoms [35]; therefore, the worsening of ulnar nerve sensory deficits in this case is presumed to be a clinical process. Because the NCS findings are not confirmed until 5 days after the onset of symptoms, a repeated NCS can help diagnose GBS [36].

The absence of abnormal findings in spinal imaging is also useful for diagnosing GBS. The differential diagnoses of complications after spinal surgery often include spinal cord or root nerve injury during positioning, hemorrhage, recurrent disc herniation, infection, displacement of an implant, vascular insults (spinal cord ischemia/injury), or pharmacological toxicity, with a reported complication rate of $4-19 \%[4,5]$. In our review, reoperation was performed in seven $(38.9 \%)$ patients $[14,15,17,18,21,22]$. Space-occupying lesions, such as an extradural hematoma or displaced operative implant, can be improved after a reoperation; however, complications might be worsened by an inappropriate operation when the origin of impairment is unclear [18]. When neurological deficits are observed after spinal surgery, GBS should be considered as a differential diagnosis when no abnormalities are present in the spine.

Although the finding of albuminocytologic dissociation in a CSF examination is helpful for the diagnosis of GBS, CSF examination is often difficult to perform after spinal surgery because of spinal changes, spinal hardware, wounds, or postoperative contamination. In this literature review, the rate of CSF examination after lumbar surgery was lower than that after cervical and thoracic vertebral surgery. The protein levels in CSF may be normal in early GBS, but they are elevated in $90 \%$ of patients by the end of the second week of symptoms [37]. Albuminocytologic dissociation also occurs 
with compression of the spinal cord [38]. This review suggests that the onset of postspinal surgery GBS is earlier than that of general GBS and that CSF testing may be performed early in the disease course. Therefore, normal or elevated CSF protein levels cannot be used to rule out or confirm a diagnosis of GBS. However, the absence of pleocytosis in CSF helps to differentiate GBS from other infectious, inflammatory, and malignant diseases [39]. If possible, CSF examination should be performed not only for the diagnosis of GBS but also for other differential diagnoses.

Our review of the literature also revealed cranial nerve involvement, autonomic dysfunction, and respiratory disorders as clinical features of GBS after spinal surgery. In this review, cranial nerve involvement was found in $50 \%$ of cases and was more common in GBS after spinal surgery than in general GBS [40]. Umer et al. [41] reported that if facial, bulbar, and neck weakness progresses within 5 days, impending respiratory failure can be expected. Autonomic dysfunction such as cardiac arrhythmia, hypertension, hypotension, diarrhea, and ileus might occur in patients with GBS [42], and the presence of these signs can trigger clinicians' recognition of GBS. In this review, gastrointestinal symptoms were the most common; in particular, diarrhea must be differentiated from infectious enteritis. This review also showed that mechanical ventilator management was needed in $50 \%$ of cases and was more frequent than in patients with general GBS [7]. Clinicians should consider the appearance of cranial neurological, autonomic, and respiratory symptoms after spinal surgery.

Prompt administration of IVIg or PE is essential for neurologic recovery in patients with GBS. These treatments should ideally be started as soon as possible; a milder benefit has been shown with treatment that is started up to 4 weeks after symptom onset [43]. Eight randomized controlled trials of the efficacy of corticosteroids for GBS showed no benefit, and treatment with oral corticosteroids was even shown to have a negative effect on outcomes [44]. In this review, only one patient required persistent ventilator management and did not improve; although the remaining patients improved, some had sequelae of weakness, numbness, facial paralysis, and dysphagia. Because no clear treatment strategy for GBS after spinal surgery has been reported, we herein propose a diagnostic flowchart for this disease (Figure 3).

\section{Conclusion}

GBS after spinal surgery is a rare neurologic complication that presents unique diagnostic challenges. Prompt diagnosis and appropriate treatment will prevent GBS from progressing to a life-threatening status. When patients exhibit unexplained weakness, areflexia, and numbness of the extremities 1-3 weeks after spinal surgery, the absence of abnormal findings in spinal imaging should be confirmed first. In patients without postoperative spinal changes, an NCS has priority regardless of whether CSF testing is available.

\section{Consent}

The patient provided informed consent, and both the patient and our hospital approved the use of the patient's clinical data.

\section{Conflicts of Interest}

The authors declare that there are no conflicts of interest.

\section{Acknowledgments}

The authors gratefully acknowledge Prof. Susumu Kusunoki, a neurologist at Kindai University, for measuring the antiganglioside antibody.

\section{References}

[1] H. J. Willison, B. C. Jacobs, and P. A. Van Doorn, "GuillainBarré syndrome," The Lancet, vol. 388, no. 10045, pp. 717-727, 2016.

[2] J. J. Sejvar, A. L. Baughman, M. Wise, and O. W. Morgan, "Population incidence of Guillain-Barré syndrome: a systematic review and meta-analysis," Neuroepidemiology, vol. 36, no. 2, pp. 123-133, 2011.

[3] H. Gensicke, A. N. Datta, P. Dill, C. Schindler, and D. Fischer, "Increased incidence of Guillain-Barré syndrome after surgery," European Journal of Neurology, vol. 19, no. 9, pp. 1239-1244, 2012.

[4] M. B. Dekutoski, D. C. Norvell, J. R. Dettori, M. G. Fehlings, and J. R. Chapman, "Surgeon perceptions and reported complications in spine surgery," Spine (Phila Pa 1976), vol. 35, no. 9 Suppl, pp. S9-s21, 2010.

[5] M. C. Willson and J. S. Ross, "Postoperative spine complications," Neuroimaging Clinics of North America, vol. 24, no. 2, pp. 305-326, 2014.

[6] Y. A. Rajabally and A. Uncini, "Outcome and its predictors in Guillain-Barré syndrome," Journal of Neurology, Neurosurgery \& Psychiatry, vol. 83, no. 7, pp. 711-718, 2012.

[7] S. R. Sudulagunta, M. B. Sodalagunta, M. Sepehrar et al., "Guillain-Barré syndrome: clinical profile and management," German Medical Science: GMS E-Journal, vol. 13, 2015.

[8] Y.-X. Zhong, G.-F. Lu, X.-L. Chen, and F. Cao, "Postoperative Guillain-Barré syndrome, a neurologic complication that must not be overlooked: a literature review," World Neurosurgery, vol. 128, pp. 347-353, 2019.

[9] T. W. Ho, B. Mishu, C. Y. Li et al., "Guillain-Barré syndrome in northern China. Relationship to Campylobacter jejuni infection and anti-glycolipid antibodies," Brain, vol. 118, no. Pt 3, pp. 597-605, 1995.

[10] S. Kuwabara, K. Ogawara, S. Misawa et al., "Sensory nerve conduction in demyelinating and axonal Guillain-Barré syndromes," European Neurology, vol. 51, no. 4, pp. 196-198, 2004.

[11] J. L. Stambough, J. G. Quinlan, and J. D. Swanson, "GuillainBarré syndrome following spinal fusion for adult scoliosis," Spine, vol. 15, no. 1, pp. 45-46, 1990.

[12] G. D. Riebel, J. G. Heller, and L. C. Hopkins, "Guillain-Barré syndrome after an operation on the spine. A case report," The Journal of Bone \& Joint Surgery, vol. 77, no. 10, pp. 1565-1567, 1995.

[13] J. Cheng, D. E. Kahn, and M. Y. Wang, "The acute motorsensory axonal neuropathy variant of Guillain-Barré 
syndrome after thoracic spine surgery," Journal of Neurosurgery: Spine, vol. 15, no. 6, pp. 605-609, 2011.

[14] D. W. Son, G. S. Song, S. K. Sung, and S. H. Kim, "GuillainBarre syndrome following spinal fusion for thoracic vertebral fracture," Journal of Korean Neurosurgical Society, vol. 50, no. 5, pp. 464-467, 2011.

[15] M. Miscusi, A. Currà, C. D. Rocca, P. Missori, and V. Petrozza, "Acute motor-sensory axonal neuropathy after cervical spine surgery," Journal of Neurosurgery: Spine, vol. 17, no. 1, pp. 82-85, 2012.

[16] F. Battaglia, A. Sevy, E. Moyse, and P.-H. Roche, "GuillainBarré syndrome following severe head trauma and spine surgery," Revue Neurologique, vol. 169, no. 2, pp. 166-168, 2013.

[17] Z. Boghani, A. D. Livingston, E. P. Simpson, P. J. Holman, and R. G. Grossman, "Acute onset of Guillain-Barré syndrome after elective spinal surgery," World Neurosurgery, vol. 84, no. 2, pp. 376-379, 2015.

[18] S.-L. Huang, H.-G. Qi, J.-J. Liu, Y.-J. Huang, and L. Xiang, "A rare complication of spine surgery: Guillain-Barré syndrome," World Neurosurgery, vol. 84, no. 3, pp. 697-701, 2015.

[19] E. Y. Chen, C. Stratton, B. Mercer, A. Hohler, T. Y. Tannoury, and C. Tannoury, "Guillain-Barré syndrome after elective spinal surgery," Journal of the American Academy of Orthopaedic Surgeons, vol. 25, no. 8, pp. 587-593, 2017.

[20] A. Rashid, S. Kurra, and W. Lavelle, "Guillain-Barré syndrome after revision lumbar surgery: a case report," Cureus, vol. 9, no. 6, Article ID e1393, 2017.

[21] N. Sahai, K. S. Hwang, and A. Emami, "Guillain-Barré syndrome following elective spine surgery," European Spine Journal, vol. 26, no. Suppl 1, pp. 6-8, 2017.

[22] J. R. Dowling and T. J. Dowling Jr., "A rare axonal variant of Guillain-Barré syndrome following elective spinal surgery," Case Reports in Orthopedics, vol. 2018, Article ID 2384969, 2018.

[23] Y. Sanpei, A. Hanazono, S. Kamada, and M. Sugawara, "Guillain-Barré syndrome and posterior reversible encephalopathy syndrome following spinal surgery," Case Reports in Neurology, vol. 11, no. 3, pp. 284-289, 2019.

[24] D.-F. Xu, B. Wu, J.-X. Wang, J. Yu, and J.-X. Xie, "Severe lumbar spinal stenosis combined with Guillain-Barré syndrome: a case report," World Journal of Clinical Cases, vol. 9, no. 5, pp. 1096-1102, 2021.

[25] P. D. Donofrio, "Guillain-Barré syndrome," CONTINUUM: Lifelong Learning in Neurology, vol. 23, no. 5, pp. 1295-1309, 2017.

[26] I. Steiner, Z. Argov, C. Cahan, and O. Abramsky, "GuillainBarre syndrome after epidural anesthesia: direct nerve root damage may trigger disease," Neurology, vol. 35, no. 10, p. 1473, 1985.

[27] N. P. Staff, J. Engelstad, C. J. Klein et al., "Post-surgical inflammatory neuropathy," Brain, vol. 133, no. 10, pp. 2866-2880, 2010.

[28] M. D. Menger and B. Vollmar, "Surgical trauma: hyperinflammation versus immunosuppression?" Langenbeck's Archives of Surgery, vol. 389, no. 6, pp. 475-484, 2004.

[29] S. Vucic, M. C. Kiernan, and D. R. Cornblath, "Guillain-Barré syndrome: an update," Journal of Clinical Neuroscience, vol. 16, no. 6, pp. 733-741, 2009.

[30] B. C. Jacobs, P. A. Van Doorn, A. P. Tio-Gillen et al., "Campylobacter jejuniinfections and anti-GM1 antibodies in Guillain-Barré syndrome,” Annals of Neurology, vol. 40, no. 2, pp. 181-187, 1996.
[31] N. Yuki, T. W. Ho, Y. Tagawa et al., "Autoantibodies to GM1b and GalNAc-GD1a: relationship to Campylobacter jejuni infection and acute motor axonal neuropathy in China," Journal of the Neurological Sciences, vol. 164, no. 2, pp. 134138, 1999.

[32] G. Ogawa, K. Kaida, M. Kuwahara, F. Kimura, K. Kamakura, and S. Kusunoki, "An antibody to the GM1/GalNAc-GD1a complex correlates with development of pure motor GuillainBarré syndrome with reversible conduction failure," Journal of Neuroimmunology, vol. 254, no. 1-2, pp. 141-145, 2013.

[33] B. R. Wakerley and N. Yuki, "Infectious and noninfectious triggers in Guillain-Barré syndrome," Expert Review of Clinical Immunology, vol. 9, no. 7, pp. 627-639, 2013.

[34] A. Uncini and S. Kuwabara, "Nodopathies of the peripheral nerve: an emerging concept," Journal of Neurology, Neurosurgery \& Psychiatry, vol. 86, no. 11, pp. 1186-1195, 2015.

[35] U. Kaur, J. S. Chopra, S. Prabhakar, K. Radhakrishnan, and S. Rana, "Guillain-Barré syndrome. A clinical electrophysiological and biochemical study," Acta Neurologica Scandinavica, vol. 73, no. 4, pp. 394-402, 1986.

[36] M. A. Albertí, A. Alentorn, S. Martínez-Yelamos et al., "Very early electrodiagnostic findings in Guillain-Barré syndrome," Journal of the Peripheral Nervous System, vol. 16, no. 2, pp. 136-142, 2011

[37] F. G. Van der Meché and P. A. Van Doorn, "Guillain-Barré syndrome and chronic inflammatory demyelinating polyneuropathy: immune mechanisms and update on current therapies," Annals of Neurology, vol. 37, no. Suppl 1, pp. S14-S31, 1995.

[38] A. Kondo, H. Yamaguchi, Y. Ishida et al., "Spontaneous spinal epidural hematoma mimicking Guillain-Barre Syndrome," Brain and Development, vol. 41, no. 4, pp. 392-395, 2019.

[39] A. D. Walling and G. Dickson, "Guillain-Barré syndrome," American Family Physician, vol. 87, no. 3, pp. 191-197, 2013.

[40] Y. Hao, W. Wang, B. C. Jacobs et al., "Antecedent infections in Guillain-Barré syndrome: a single-center, prospective study," Annals of Clinical and Translational Neurology, vol. 6, no. 12, pp. 2510-2517, 2019.

[41] S. R. Umer, Q. Nisa, M. Kumari, S. Abbas, S. A. Mahesar, and N. N. Shahbaz, "Clinical features indicating the need for mechanical ventilation in patients with guillain barre syndrome," Cureus, vol. 11, no. 8, Article ID e5520, 2019.

[42] S. Kuwabara, "Guillain-barr?? syndrome," Drugs, vol. 64, no. 6, pp. 597-610, 2004.

[43] R. A. C. Hughes, E. F. M. Wijdicks, R. Barohn et al., "Practice parameter: immunotherapy for Guillain-Barre syndrome: report of the quality standards subcommittee of the American academy of neurology," Neurology, vol. 61, no. 6, pp. 736-740, 2003.

[44] R. A. Hughes, A. V. Swan, J. C. Raphaël, D. Annane, R. Van Koningsveld, and P. A. Van Doorn, "Immunotherapy for Guillain-Barré syndrome: a systematic review," Brain, vol. 130, no. Pt 9, pp. 2245-2257, 2007. 